\title{
Molecular epidemiology of chronic pulmonary colonisation by Pseudomonas aeruginosa in cystic fibrosis
}

\author{
J. BOUKADIDA, M. DE MONTALEMBERT, G. LENOIR $\dagger$, P. SCHEINMANN $\ddagger$, M. VÉRON \\ and P. BERCHE*
}

Laboratoire de Microbiologie, †Service de Pédiatrie Générale, $¥$ Service de Pneumologie et Allergologie, Hôpital Necker-Enfants Malades, 149 rue de Sèvres, 75015 Paris, France

\begin{abstract}
Summary. The epidemiology of pulmonary colonisation by Pseudomonas aeruginosa was studied in 21 patients with cystic fibrosis (CF) by field inversion gel electrophoresis. DraIDNA restriction patterns were analysed for 187 P. aeruginosa isolates from these patients. The results revealed that the strains present in individual patients varied during the course of chronic colonisation; the emergence of new strains often was associated with periods of antibiotic therapy. Patients often were colonised by more than one strain (two or three strains were present in $54 \%$ of the patients) and the strains obtained from unrelated patients were highly heterogeneous, in contrast to those isolated from a pair of twins. These results demonstrate the heterogeneity and variability of $P$. aeruginosa isolates in the pulmonary flora of chronically infected CF patients.
\end{abstract}

\section{Introduction}

Pseudomonas aeruginosa is a major opportunist pathogen in patients with cystic fibrosis $(\mathrm{CF}) .^{\mathbf{1}, 2}$ Pulmonary infection by $P$. aeruginosa in these patients is refractory to treatment with antibiotics and ultimately leads to clinical deterioration and death. ${ }^{1}$ Surprisingly, little is known about the epidemiology and transmission of $P$. aeruginosa in CF patients, particularly as regards (i) the diversity of strains present among different chronic carriers followed at the same medical centre, (ii) the variability and the multiplicity of strains present in the individual patients, (iii) the influence of antibiotic therapy on the emergence of new strains, and (iv) the impact of person-to-person and environmental exposure on chronically infected patients. This lack of information stems from the limits of conventional typing methods based on serotype, phage type or pyocin production. These methods are unsuitable for many of the mucoid strains isolated from CF patients. ${ }^{3}$ New typing methods based on genome analysis, such as pulsed field electrophoresis, may be more appropriate for epidemiological studies on $P$. aeruginosa isolates from CF patients. Pulsed field electrophoresis allows the separation of large nucleic-acid fragments (50$9000 \mathrm{~kb}$ ), produced by digestion of bacterial DNA with endonucleases that cut at infrequent restriction sites. $^{4,5}$ The restriction patterns produced appear specific to individual strains and have already been

Received 8 Jan. 1992; revised version accepted 30 April 1992.

* Correspondence should be sent to Dr P. Berche. applied to the analysis of the epidemiology of bacterial infections, ${ }^{6,7}$ including those in the lungs of $\mathrm{CF}$ patients. ${ }^{8-10}$ In the present study this method was used to study the epidemiology of $P$. aeruginosa in chronically infected CF patients.

\section{Patients and methods}

\section{Patients}

Twenty-one patients, 11 female and 10 male, aged between 4 and 22 years, were studied. They comprised 19 unrelated individuals and one pair of twins. All were followed in the CF Centre of the Hôpital Necker-Enfants Malades. All these patients were colonised by $P$. aeruginosa, some for $<1$ year, but most for several years (fig. 1). Exacerbations of their infections were treated by 2-4-week courses of various antibiotic combinations, generally ceftazidime-tobramycin, ciprofloxacin-tobramycin or imipenem-amikacin. Patients 12 and 20 died during the study, the former shortly after heart-lung transplant. Most patients included in this study were regularly hospitalised, usually for periods of $c .2$ weeks, in a paediatric ward of our hospital. This unit comprised 10 single bedrooms, two double-bedrooms, and two fourbedded rooms, each equipped with sinks. Patients shared the same shower, bath and toilet facilities. Infected $\mathrm{CF}$ patients were not segregated from one another and shared many common activities including meals, games, toys and hospital school, thus allowing frequent and close person-to-person exposure. 


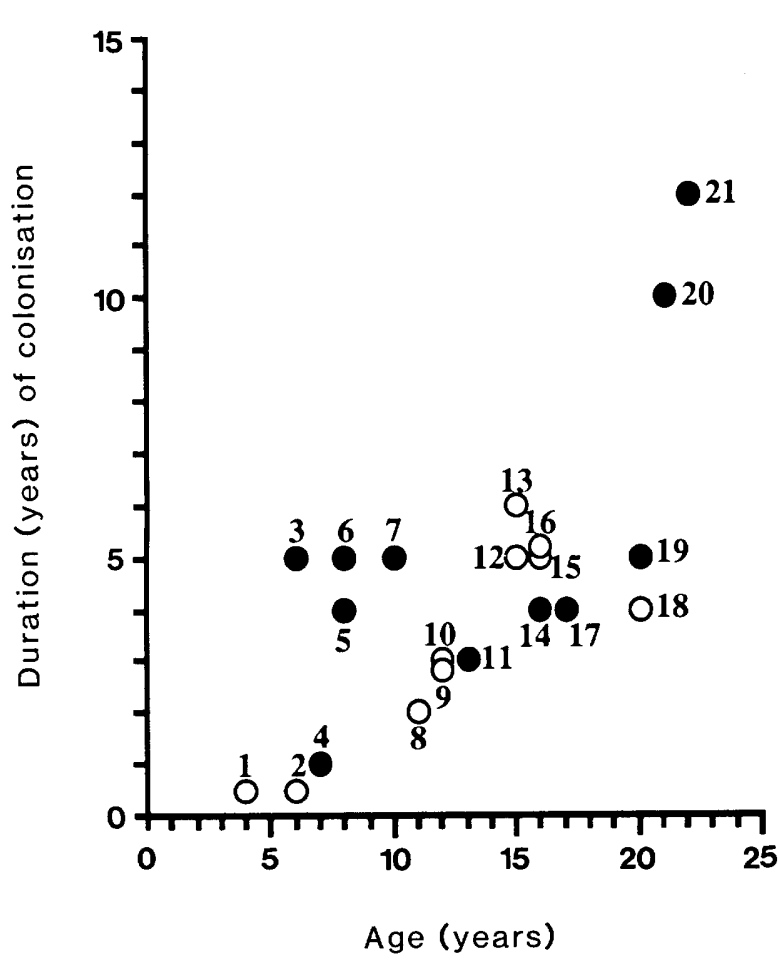

Fig. 1. Duration of pulmonary colonisation with $P$. aeruginosa in 21 CF patients: $O$ male; female.

\section{Bacteriological methods}

Selective Cetrimide Medium (Diagnostics Pasteur, Marne la Coquette, France) was used for culture of respiratory specimens. Most of the $P$. aeruginosa isolates from the sputum of the $\mathrm{CF}$ patients initially produced mucoid colonies. Sometimes, non-mucoid clones segregated, which variously had "fried-egg", "smooth" or "dwarf" colony morphology. Bacteria were identified with the API-20NE system (API System, La Balmes-les-Grottes, France). Serotyping was performed with 16 monospecific sera directed against $\mathrm{O}$ antigens (Diagnostics Pasteur). More than half of the isolates could not be serotyped. Antibiotic susceptibility was studied by the agar diffusion method on Mueller-Hinton Medium (Diagnostics Pasteur). Separated colonies were grown and stored at $-70^{\circ} \mathrm{C}$ in LabLenco Medium (Oxoid) until electrophoretic analysis.

\section{Field inversion gel electrophoresis (FIGE)}

Bacterial DNA was analysed by field inversion gel electrophoresis (FIGE). This pulsed field electrophoresis technique was based on that of Grothues et al. ${ }^{9}$ and allowed good separation of DNA fragments of $50-1000 \mathrm{~kb}$. Briefly, bacteria from a single colony were grown in tryptone-water to logarithmic phase, harvested, and then resuspended to an optical density of 1.5 at a wavelength of $650 \mathrm{~nm}$. The bacterial suspension was mixed with an equal volume of Incert Agarose (FMC BioProducts, Rockland, ME, USA), to form an agar insert, then incubated for $48 \mathrm{~h}$ at $55^{\circ} \mathrm{C}$ with 0.5 M EDTA (Sigma), pH 9, sarcosine (Sigma) $1 \% \mathrm{w} / \mathrm{v}$ and proteinase $\mathrm{K}$ (Appligene, Illkirch,
France) $1 \mathrm{~g} / \mathrm{L}$. Subsequently, the inserts were washed extensively: twice for $20 \mathrm{~min}$ each with TE buffer $(0.1 \mathrm{~m}$ Tris, $0.01 \mathrm{~m}$ EDTA, pH 7.5) then with $0.01 \mathrm{M}$ phenylmethyl sulphonyl fluoride (Sigma); next with TE buffer for $1 \mathrm{~h}$ at $37^{\circ} \mathrm{C}$ and finally with $1 \mathrm{~mm}$ Tris$\mathrm{HCl}$ buffer, $\mathrm{pH} 7 \cdot 5$, overnight at $4^{\circ} \mathrm{C}$. Since the GC mol \% of $P$. aeruginosa is $67.2 \%,{ }^{2}$ restriction endonucleases recognising AT-rich restriction sites were used (DraI, XbaI, SpeI, SspI, NheI). The best results were obtained with $X b a I$ and $D r a I$, which cut TCTAGA and TTTAAA sites respectively. In this report, only the DraI restriction patterns are presented. Purified DNA was digested overnight by $\operatorname{DraI}$ (30 IU) (Appligene). The fragments were separated by FIGE

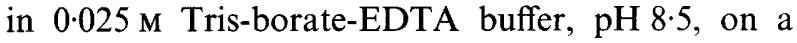
Rotaphore 10035 Apparatus (Biometra, Göttingen, Germany), run at $10^{\circ} \mathrm{C}$ and a field strength of $5 \mathrm{~V} / \mathrm{cm}$ for $96 \mathrm{~h}$. The forward: reverse ratio was $3: 1$. The gels were stained with ethidium bromide. Controls included DNA from $\lambda$ phage (FMC BioProducts) and DNA from $P$. aeruginosa ATCC 27853, prepared by the same method as described above. Restriction profiles were considered to be identical even if they differed by one or two bands. Such minor differences most probably result from point mutations at restriction sites. ${ }^{9}$ As controls, we tested 43 wild-type non-mucoid $P$. aeruginosa isolates from opportunist infections in non CF-patients. These isolates all gave different DraI restriction patterns (data not shown), confirming the high genomic diversity amongst $P$. aeruginosa strains already observed by FIGE analysis. ${ }^{9}$

\section{Results}

\section{Analysis of serial P. aeruginosa isolates from $C F$ patients}

The $P$. aeruginosa isolates cultured from 10 to 13 sequential sputum samples from each of three patients (nos. 12, 17 and 20) were examined. Each of these patients was between 15 and 20 years old and had been colonised for 4-10 years. With some samples, more than one colony type was seen on the culture plates. In such cases, each colony type was analysed separately and, in total, 68 isolates were examined by FIGE of DraI-digested DNA (fig. 2).

The first samples from Patient 20 contained a single strain. This strain was rapidly replaced by another strain, which remained dominant in the patient's sputum during the next 15 months, despite repeated antibiotic therapy. The other two patients frequently harboured more than one strain at a time (e.g., specimen 4 from Patient 12). Shortly after heart-lung transplantation, Patient 12 was colonised with a new strain, never previously isolated from this individual. The emergence of new strains was usually associated with periods of antibiotic therapy, which failed to eradicate $P$. aeruginosa strains from the sputum of these patients. No particular antibiotic therapy was 
Patient 12

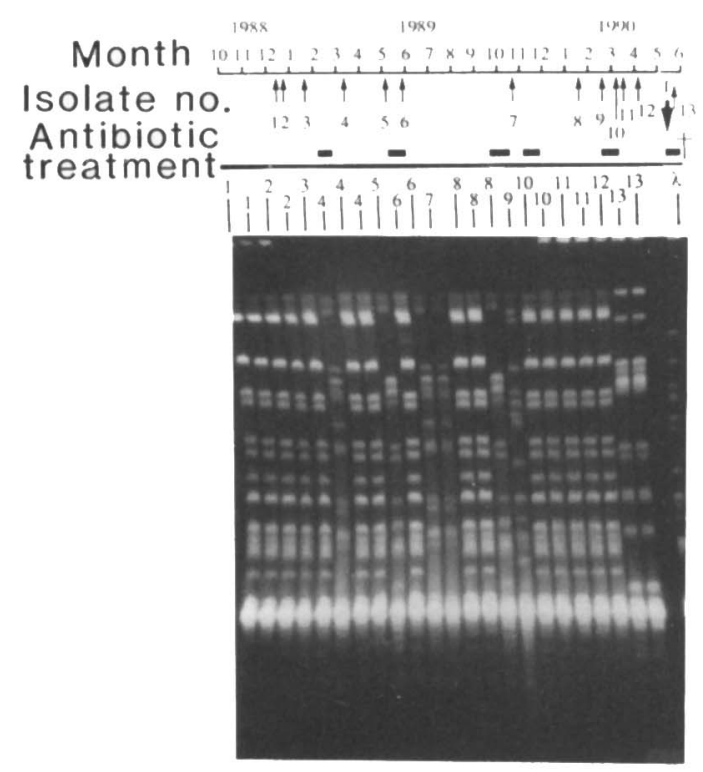

Patient 17
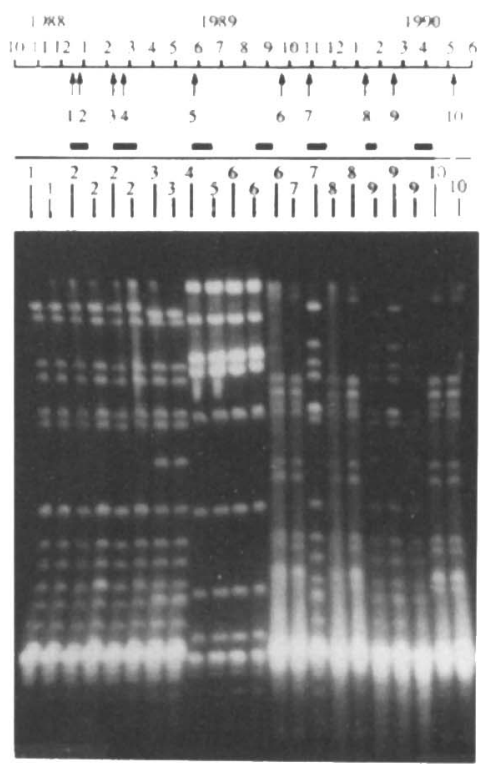

Patient 20

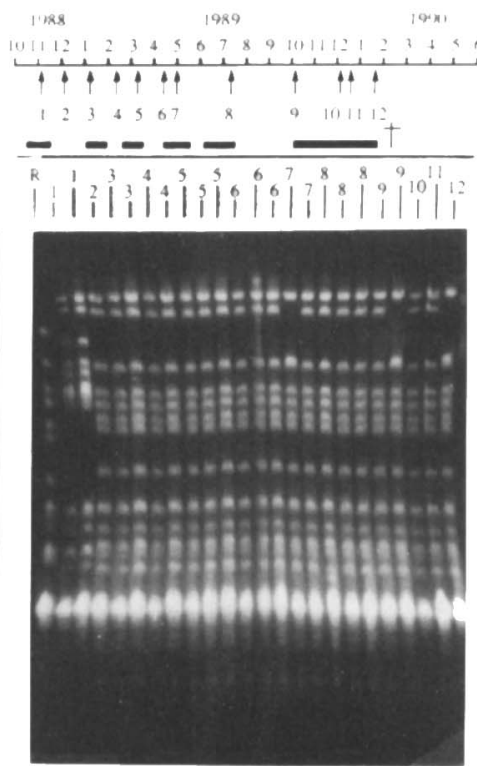

Fig. 2. Dral-restriction patterns of 68 mucoid strains of $P$. aeruginosa sequentially isolated from three CF patients. The specimens from each patient are numbered in order of collection, and the dates of collection are indicated in the upper part of the figure. One to four colonial phenotypes were studied for every specimen. $\mathrm{T}$, heart-lung transplantation; $\dagger$, death; $\mathrm{R}$, reference strain ATCC $27853 ; \hat{\lambda}$, concatemer phage $\lambda$.

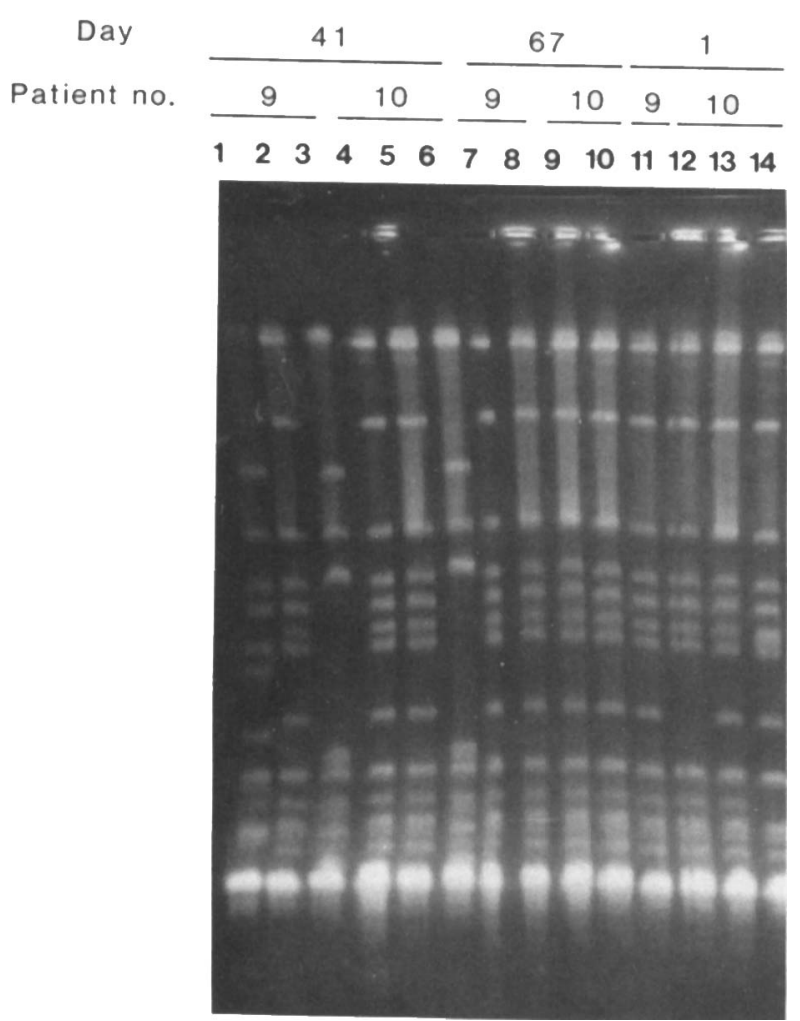

Fig. 3. FIGE restriction patterns of $14 P$. aeruginosa isolates from twins with CF. Day 1: lane 11, twin No. 9;12-14, twin No. 10. Day 41: lanes 1-3, twin No. 9; 4-6, twin No. 10. Day 69: lanes 7-8, twin No. $9 ; 9-10$, twin No. 10 .

associated with the emergence of new strains in these three patients.

\section{Heterogeneity of $P$. aeruginosa isolates from $C F$ patients}

FIGE was used to analyse a total of 187 mucoid $P$. aeruginosa isolates from the 21 chronically infected
Table. Colonisation with multiple $P$. aeruginosa strains in CF patients

\begin{tabular}{|c|c|c|c|c|c|}
\hline \multirow{2}{*}{$\begin{array}{l}\text { Duration of } \\
\text { colonisation by } \\
P . \text { aeruginosa }\end{array}$} & \multirow{2}{*}{$\begin{array}{l}\text { Patient* } \\
\text { Nos. }\end{array}$} & \multirow{2}{*}{$\begin{array}{c}\text { Number } \\
\text { of } \\
\text { samples }\end{array}$} & \multicolumn{3}{|c|}{$\begin{array}{c}\text { Number of } P \text {. aeruginosa } \\
\text { strains detected } \dagger\end{array}$} \\
\hline & & & 1 & 2 & 3 \\
\hline$<1$ year & 1,2 & 2 & 2 & 0 & 0 \\
\hline $3-5$ years & $\begin{array}{c}7,9-13 \\
16,17,19\end{array}$ & 15 & 5 & 8 & 2 \\
\hline$>10$ years & 20,21 & 4 & 3 & 1 & 0 \\
\hline Total & 13 & 21 & 10 & 9 & 2 \\
\hline
\end{tabular}

* See reference numbers in fig. 1 .

$\dagger$ Three to six isolated colonies of $P$. aeruginosa were tested by FIGE for each specimen, except for Patient I from whom 14 clones were tested. In total 83 isolates were examined.

CF patients. The strains isolated from 19 unrelated patients all appeared different to one another (not shown). In contrast, the restriction patterns of 14 serial isolates cultured from a pair of $\mathrm{CF}$ twins were identical during a 2-month period (fig. 3). The twins (Patients 9 and 10), aged 12, had been colonised at the same time, 3 years before the study. At the start of the study, both carried the same single strain. After a 2-week period of antibiotic therapy (day 41), they were found to be colonised by two or three different strains, still including the pre-therapy strain, which again predominated by day 67 of the study (fig. 3).

\section{Multiple $P$. aeruginosa isolates from individual patients}

The existence of multiple $P$. aeruginosa strains in sputum specimens from CF patients was demonstrated by analysing, with FIGE, between three and six colonies from each of 21 samples obtained from 13 
colonised CF patients. These comprised two individuals colonised for $<1$ year, nine infected for 3-5 years, and two colonised for $>10$ years (table); 83 clones were analysed. In seven $(54 \%)$ of the 13 patients, two or three strains dominated, two patients $(15 \%)$ being infected by three distinct strains. The two recently colonised patients ( $<1$ year), probably harboured only single strains; six to 14 colonies examined were identical within each of these individuals.

\section{Discussion}

When FIGE was used to study the epidemiology of chronic pseudomonal colonisation in CF patients, it was found that the $P$. aeruginosa strains isolated from sputum varied during chronic infection. Analysis of 68 serial isolates from three patients over periods of 15-18 months revealed that the dominant strain could vary, that new strains could emerge and sometimes previous strains could re-emerge after periods in which they were not apparent in the flora (fig. 2). Administration of antibiotics, which always failed to eradicate $P$. aeruginosa infection, often led to changes to the strains isolated, probably caused by the selection of strains that were previously present in low numbers. However, we did not find any change to the strain present in Patient 20, regardless of treatment. A similar result was reported for another patient. followed over a 2 -year period by Pasloske et al. ${ }^{11}$ by a DNA probing method.

Previous studies, based on examination of colonial phenotypes or classical markers, have disagreed on whether pulmonary colonisation by $P$. aeruginosa in $\mathrm{CF}$ patients is homogeneous, or heterogeneous. ${ }^{11^{15}} \mathrm{In}$

\section{References}

1. Fick RB. Pathogenesis of the Pseudomonas lung lesion in cystic fibrosis. Chest 1989: 96:158-164.

2. Hoiby N. Rosendal K. Epidemiology of Pseudomonas aeruginosa infection in patients treated at a cystic fibrosis centre. Acta Pathol Microbiol Siand Sect B 1980;88: 125131 .

3. Pitt TL. Epidemiological typing of Psendomonas aeruginosa. Eur J Clin Microbiol Infect Dis 1988: 7: 238--247.

4. Carle GF, Franck M. Olson MV. Electrophoretic separations of large DNA molecules by periodic inversion of the electric field. Science 1986: 232:65-68.

5. Schwartz DC. Cantor CR. Separation of yeast chromosomesized DNAs by pulsed field gradient gel electrophoresis. Cell 1984:37:67-75

6. Allardet-Servent A. Bourg G. Ramuz M, Pages M, Bellis M, Roizes G. DNA polymorphism in strains of the genus Brucella. I Bacteriol 1988: 170:4603 4607.

7. Allardet-Servent A. Bouziges $N$, Carles-Nurit MJ. Bourg G. Gouby A. Ramuz M. Use of low-frequency-cleavage restriction endonucleases for DNA analysis in epidemiological investigations of nosocomial bacterial infections. J Clin Microbiol 1989: 27:2057-2061.

8. Grothues D. Koopmann U. Von Der Hardt H. Tümmler B Genome fingerprinting of Pseudomonas aeruginosa indicates colonization of cystic fibrosis siblings with closely related strains. J Clin Microbiol 1988: 26: 1973-1977.

9. Grothues D. Tümmler B. Genome analysis of Pseudomonas this study, 83 strains from 21 sputum samples obtained from 13 patients were examined. Seven of $13(54 \%)$ patients were colonised by more than one strain. Grothues et al. ${ }^{8}$ also used FIGE, and found that $37 \%$ of patients were infected by more than one strain. Since we analysed only three to six colonies from the sputum of each patient, it is probable that we failed to detect the presence of multiple strains in many more patients.

Based on DraI-restriction patterns, the strains isolated from 19 unrelated patients were highly heterogeneous, in contrast to strains isolated from a pair of twins. Similar results were reported by Grothues et al. ${ }^{8}$ The diversity of strains isolated from unrelated patients who had been followed with periodic hospitalisation, for several years in the same medical centre with repeated contacts, argues against crossinfection. This is in contrast to the behaviour of $P$. cepacia, with which cross-infection has been reported. ${ }^{16}$ It has been reported that non-infected $\mathrm{CF}$ patients are resistant to colonisation by mucoid strains of $P$. aeruginosa ${ }^{17}$ Although the environmental source of primary colonisation by $P$. aeruginosa remains poorly understood, ${ }^{18.19}$ our data suggest that it is most likely from the wide variety of environmental sources where $P$. aeruginosa can occur, thus explaining the heterogeneity of strains.

In conclusion, in addition to plasmid fingerprinting ${ }^{20,21}$ and to DNA probing, ${ }^{11,22}$ pulsed field electrophoresis is a powerful epidemiological tool for the study of colonisation by $P$. aeruginosa in $\mathrm{CF}$ patients.

We thank Marie-Laurence Fourneaux for typing the manuscript and preparing the illustrations. This study was supported by a grant 1990 from the "Association Française de Lutte contre la Mucoviscidose" (AFLM). aeruginosa by field inversion gel electrophoresis. FEMS Microbiol Lett $1987 ; 48$ : 419-422.

10. Boukadida J, de Montalembert M, Gaillard JL et al. Outbreak of gut colonization by Pseudomonas aeruginosa in immunocompromised children undergoing total digestive decontamination: analysis by pulsed-field electrophoresis. $J$ Clin Microbiol 1991; 29: 2068-2071.

11. Pasloske BL, Joffe AM, Sun Q et al. Serial isolates of Pseudomonas aeruginosa from a cystic fibrosis patient have identical pilin sequences. Infect Immun $1988 ;$ 56; 665-672.

12. Høiby N, Pedersen SS. Estimated risk of cross-infection with Pseudomonas aeruginosa in Danish cystic fibrosis patients. Acta Paediatr Scand 1989; 78: 395-404.

13. Horrevorts AM, Borst J, Puyk RJT et al. Ecology of Pseudomonas aeruginosa in patients with cystic fibrosis. $J$ Med Microbiol 1990; 3: 119-124.

14. Seale TW, Thirkill H, Tarpay M, Flux M, Rennert OM. Serotypes and antibiotic susceptibilities of Pseudomonas aeruginosa isolates from single sputa of cystic fibrosis patients. J Clin Microbiol 1979; 9: 72-78.

15. Thomassen MJ, Demko CA, Boxerbaum B, Stern RC, Kuchenbrod PJ. Multiple isolates of Pseudomonas aeruginosa with differing antimicrobial susceptibility patterns from patients with cystic fibrosis. J Infect Dis 1979; 140:873 880.

16. Lipuma JJ, Dasen SE, Nielson W, Stern RC, Stull TL. Personto-person transmission of Pseudomonas cepacia between patients with cystic fibrosis. Lancet 1990; 336: 10941096. 17. Speert DP, Lawton D, Damm S. Communicability of Pseudo- 
monas aeruginosa in a cystic fibrosis summer camp. $J$ Pediatr 1982; 101: 227-229.

18. Kelly NM, Tempany E, Falkiner FR, Fitzgerald MX, O'Boyle C, Keane CT. Does Pseudomonas cross-infection occur between cystic fibrosis patients? Lancet 1982; 2: 688-690.

19. Zimzoff J, Høiby $\mathrm{N}$, Rosendant $\mathrm{K}$ et al. Epidemiology of Pseudomonas aeruginosa infection and the role of contamination of the environment in a cystic fibrosis clinic. $J$ Hosp Infect $1983 ; 4: 31-40$.

20. Poh CL, Yap EH, Tay L, Bergan T. Plasmid profiles compared with serotyping and pyocin typing for epidemiological surveillance of Pseudomonas aeruginosa. J Med Microbiol $1988 ; 25: 109-114$

21. Tenover FC. Plasmid fingerprinting: a tool for bacterial strain identification and surveillance of nosocomial and community-acquired infections. Clin Lab Med 1985; 5: 413436.

22. Ogle JW, Janda JM, Woods DE, Vasil ML. Characterization and use of a DNA probe as an epidemiological marker for Pseudomonas aeruginosa. J Infect Dis 1987; 155: 119-126. 